\title{
Producción de biodiesel a partir de residuos grasos animales por vía enzimática
}

\author{
Por Ivanna Rivera, Gerardo Villanueva y Georgina Sandoval*
}

\author{
Centro de Investigación y Asistencia en Tecnología y Diseño del Estado de Jalisco (CIATEJ), \\ 44270 Guadalajara, Jalisco, México. \\ ( ${ }^{\star}$ Autor para correspondencia: gsandoval@ ciatej.net.mx / georgina@ confluencia.net)
}

\begin{abstract}
RESUMEN
Producción de biodiesel a partir de residuos grasos animales por vía enzimática

Grasas animales extraídas directamente de residuos orgánicos de un matadero municipal fueron empleadas con éxito en la reacción de transesterificación como materia prima alternativa de bajo costo para la producción de biodiesel. Las reacciones de transesterificación se realizaron por vía enzimática con dos lipasas comerciales N435 y RM IM. De las dos enzimas probadas, la enzima N435 fue la más eficiente tanto en el sistema libre de solvente como en el sistema con terbutanol como solvente. En ambos sistemas el exceso de etanol fue la mejor condición para la reacción alcanzando conversiones de $80 \%$ en 48 horas en el caso del sistema libre de solvente y $65 \%$ en 24 horas en el sistema con terbutanol.
\end{abstract}

PALABRAS CLAVE: Biodiesel - Etil ésteres - Lipasa Residuos grasos - Solvente - Transesterificación.

\section{SUMMARY}

Biodiesel production from animal grease wastes by enzymatic catalysis

Animal grease wastes were successfully used in the transesterification reaction as an alternative raw material for biodiesel production. Transesterification reactions were performed using an enzymatic catalysis with two commercial lipases N435 and RM IM. N435 was the most efficient in a solvent free system as well as in the system with terbuthanol. In both systems the excess of ethanol turned out to be beneficial for the reaction obtaining conversions of $80 \%$ in 48 hours and $65 \%$ in 24 hours.

KEY-WORDS: Biodiesel - Ethyl esters - Grease waste Lipase - Solvent free system - Transesterification.

\section{INTRODUCCIÓN}

El biodiesel se define como una mezcla de monoacilésteres de ácidos grasos de cadena larga de origen vegetal o animal, para su uso en combustión. Además este biocombustible constituye una alternativa viable para la obtención de energía limpia y renovable. (Knothe y Dunn, 1996).

Sin embargo, su precio en promedio en el mercado es el doble del precio del diesel fósil (Balat y Balat, 2008; Canakci y Sanli, 2008). Adicionalmente, algunos estudios (Haas et al., 2006) han determinado que la materia prima representa apro- ximadamente entre $75 \%$ y $88 \%$ del costo total de producción de este biocombustible por lo que es imperativo reducir estos costos para obtener un producto competitivo en el mercado. Para ello, una opción prometedora es encontrar alternativas a las materias primas usadas tradicionalmente que actualmente son aceites vegetales vírgenes o refinados.

Entre las materias primas alternativas, destacan los residuos grasos animales y vegetales, los cuales son una materia prima ideal a bajo costo. Además se ha demostrado que existe una buena disponibilidad, por ejemplo en USA se tiene una disponibilidad estimada de 470 millones de toneladas (Canakci, 2005). Estos residuos podrían ser utilizados como materia prima para biodiesel, evitando los debates éticos de utilizar cultivos y tierras que se destinan para fines alimenticios.

Convencionalmente el biodiesel se produce utilizando catalizadores básicos (Meher et al., 2006), los cuales presentan problemas de recuperación del catalizador y corrosión. Por otra parte, la catálisis enzimática se ha explorado recientemente con buenos resultados (Al-Zuhair, 2005; Dizge y Keskinler, 2008; Du, 2005; Royon et al., 2007); demostrando ser un sistema que proporciona grandes ventajas en el proceso como: mayor seguridad, pueden ser usadas materias primas de menor calidad, el producto y subproducto se obtienen con mayor grado de pureza y los procesos enzimáticos son generalmente más limpios y ecológicos.

Además, en estudios recientes se han probado residuos grasos vegetales en la producción de biodiesel. Sin embargo hay muy pocos estudios sobre residuos grasos animales, los cuales al tener mayor contenido de ácidos grasos saturados, proporcionan mayor estabilidad al producto final, independientemente del catalizador empleado, aunque puede aumentar la temperatura de fusión del biodiesel obtenido, lo que puede ser una desventaja bajo algunos climas (Lee et al., 2002), más no así en el caso de climas tropicales.

En este trabajo se estudia el efecto de la relación molar y el uso del terbutanol como solvente sobre la reacción de transesterificación sobre residuos grasos animales extraídos de los residuos orgánicos de un matadero municipal. Para ello, se caracterizaron las materias primas y se llevaron a 
cabo los estudios de los efectos estadísticamente significativos de estos factores sobre la velocidad inicial de reacción y la conversión.

\section{MATERIALES Y MÉTODOS}

\subsection{Materiales}

Las lipasas RM IM (lipasa de Rhizomucor miehei inmovilizada soporte hidrofílico) y N435 (lipasa B de Candida antarctica inmovilizada en soporte hidrofóbico), fueron una generosa donación de Novozymes, los residuos grasos animales fueron obtenidos del matadero municipal de la ciudad de San Luis Potosí (México), el hexano HPLC fue marca Karal y todos los demás solventes utilizados fueron grado reactivo.

\subsection{Extracción de las grasas}

Las muestras obtenidas de residuos orgánicos de res y de cerdo (conteniendo porciones visibles de grasa) son cortadas en porciones pequeñas para posteriormente extraer la grasa mediante calentamiento en estufa a una temperatura de $50^{\circ} \mathrm{C}$ durante 24 horas. El líquido obtenido se hace pasar por una gasa para evitar la presencia de residuos sólidos. Finalmente se almacena a -20 grados centígrados para su posterior uso.

\subsection{Caracterización de las grasas}

\section{Perfil de ácidos grasos}

Para la obtención del perfil de ácidos grasos se utiliza el método de trifloruro de boro y para el análisis cromatográfico se emplea un cromatógrafo de gases Agilent 6980, equipado con una columna HP 23 y cuyo método opera bajo las siguientes condiciones temperatura inicial del horno: $200{ }^{\circ} \mathrm{C}$, gradiente: $25^{\circ} \mathrm{C} / \mathrm{min}$ hasta $180^{\circ} \mathrm{C}$ y mantener por 30 min además $5^{\circ} \mathrm{C} / \mathrm{min}$ hasta $250^{\circ} \mathrm{C}$ durante $15 \mathrm{~min}$., flujo de helio $2 \mathrm{ml} / \mathrm{min}$., flujo de aire: $400 \mathrm{ml} / \mathrm{min}$, flujo de $\mathrm{H}_{2}: 40 \mathrm{ml} / \mathrm{min}$, volumen de inyección: 0.5 $\mu L$. ., makeup: $30 \mathrm{ml} / \mathrm{min}$, relación split: 100/1. Para la derivatización se adicionan 300 mg de muestra a un matraz con perlas de ebullición desengrasadas y $4 \mathrm{~mL}$ de $\mathrm{NaOH} 0.5 \mathrm{~N}$. Se conecta un refrigerante y se coloca en baño maría a $75^{\circ} \mathrm{C}$ por 12 min. Pasado este tiempo se añade por la parte superior del refrigerante $5 \mathrm{~mL}$ de trifluoruro de boro y se deja en el baño otros 2 min. Posteriormente, adicionar 3 $\mathrm{mL}$ de n-heptano y se mantiene en el baño $1 \mathrm{~min}$. El matraz se retira del sistema y se tapa para enfriarlo con agua corriente agitando al mismo tiempo. Se agregan $20 \mathrm{~mL}$ de solución saturada de $\mathrm{NaCl}$ para llevar la fase orgánica al cuello del matraz. Mediante una pipeta Pasteur, ayudado de una perilla se recoge la fase heptánica tomando 1 mililitro del extracto para inyectar la muestra en el cromatógrafo.
La relación molar grasa:etanol fue calculada en base a la composición en ácidos grasos encontrada. La masa molecular de la mezcla reaccional se cálculo en $936 \mathrm{~g} / \mathrm{mol}$.

\section{Índice de yodo}

El índice de peróxido o índice de yodo se determinó usando el método 28.022-28.023 de AOAC (1984).

\section{Índice de acidez}

El contenido de ácidos grasos libres (AGL) se determinó por titulación alcalina de acuerdo al método Ca 5a-40 de la AOCS (1989) y su resultado es expresado como por ciento de ácido oleico.

\subsection{Reacciones de transesterificación}

Las reacciones de transesterificación se llevaron a cabo en un volumen de $5 \mathrm{~mL}$ de. La mezcla de reacción constaba de $0.8 \mathrm{M}$ de una mezcla $50 \%$ grasa de cerdo y $50 \%$ grasa de res, la proporción correspondiente de etanol para alcanzar una relación molar $1: 1$ y $1: 4$ triglicérido: etanol y $100 \mathrm{mg}$ de lipasa. Las condiciones de operación en las que se llevaron a cabo las reacciones fueron $45^{\circ} \mathrm{C}$ y una agitación magnética de 300 rpm.

Las reacciones de transesterificación en terbutanol se llevaron a cabo en un volumen de $5 \mathrm{~mL}$ de la mezcla reaccional que constaba de $0.2 \mathrm{M}$ de una mezcla $50 \%$ grasa de cerdo y $50 \%$ grasa de res, la proporción correspondiente de etanol para alcanzar una relación molar 1:1 y 1:4 triglicérido: etanol y $100 \mathrm{mg}$ de lipasa. Las condiciones de operación en las que se llevaron a cabo las reacciones fueron $45^{\circ} \mathrm{C}$ y una agitación magnética de $300 \mathrm{rpm}$.

\subsection{Análisis cromatográficos y estadísticos}

$100 \mu \mathrm{L}$ de muestra fueron diluidos en $900 \mu \mathrm{L}$ de hexano HPLC y analizadas por cromatografía de gases con una columna HP5 bajo las siguiente condiciones: Temperatura inicial del horno: $200^{\circ} \mathrm{C}$., gradiente: $5^{\circ} \mathrm{C} / \mathrm{min}$, hasta $300{ }^{\circ} \mathrm{C}$ y mantener por 13 min., Flujo de helio $2 \mathrm{ml} / \mathrm{min}$., Flujo de aire: 400 $\mathrm{ml} / \mathrm{min}$, Flujo de $\mathrm{H}_{2}: 40 \mathrm{ml} / \mathrm{min}$, Volumen de inyección: $0.2 \mu$ L.Makeup: $30 \mathrm{ml} / \mathrm{min}$, Relación split: 50/1.

Todas las determinaciones fueron llevadas a cabo por duplicado. El cálculo del porcentaje de conversión en las reacciones de transesterificación se llevo a cabo mediante la siguiente ecuación:

$\%$ Conversión $=\frac{\left(\mathrm{mM} \text { totales de etil ésteres }{ }^{*} 100\right)}{\left(3^{*} \mathrm{mM} \text { triglicérido }\right)}(1)$

El análisis de los datos experimentales se llevo a cabo por medio del software Statgraphics ${ }^{\mathrm{MR}}$. EI diseño experimental utilizado fue un $2^{k}$ con una réplica. Los tres factores estudiados fueron: el biocatalizador, la presencia de solvente y el radio 
molar. Con dos variables de respuesta: \% de conversión y velocidad inicial de reacción.

\section{RESULTADOS Y DISCUSIÓN}

\subsection{Caracterización de las grasas}

La determinación de índice de peróxido permite identificar el nivel de oxidación, presente en grasas y aceites, lo cual es un indicativo de la calidad de los mismos. Analizados los índices de peróxido obtenidos en los residuos grasos (Tabla 1), se observa que en nuestro caso son menores a los encontrados en la literatura, lo que es indicativo de poca oxidación (Badui, 1993). Esto puede ser debido a que las grasas son extraídas directamente de los residuos orgánicos y que se encuentran protegidas de la oxidación por los tejidos, lo cual representa una ventaja.

El índice de acidez (Tabla 1), nos permite determinar la presencia de ácidos grasos libres en la muestra. Es importante mencionar que en el caso de la catálisis enzimática la presencia de ácidos grasos libres no afecta el rendimiento de la reacción, ya que las enzimas son capaces de llevar a cabo la reacción de esterificación directa a partir de ácidos grasos libres (Canakci, 2005; Lee et al., 2002). Esto es una ventaja frente al proceso clásico en el cual se emplean catalizadores básicos donde la presencia de ácidos libres en las grasas o

Tabla 1

Características de las grasas

\begin{tabular}{lcc}
\hline \multicolumn{1}{c}{ Determinación } & $\begin{array}{c}\text { Grasa } \\
\text { de res }\end{array}$ & $\begin{array}{c}\text { Grasa } \\
\text { de cerdo }\end{array}$ \\
\hline $\begin{array}{l}\text { Índice de acidez } \\
(\% \text { acido oleico })\end{array}$ & 4.8 & 0.4 \\
Índice de peróxido $(\mathrm{meq} / \mathrm{kg})$ & 1.0 & 17.9 \\
\hline
\end{tabular}

aceites, así como un alto nivel de oxidación, afectan de manera significativa los rendimientos de la reacción.

En el perfil de ácidos grasos que se presenta en la Tabla 2, se observa la presencia de alrededor de $50 \%$ de ácidos grasos saturados en la grasa de cerdo y alrededor de $70 \%$ en la de res, lo cual es una de las principales diferencias con respecto a la composición de los aceites vegetales. Los residuos grasos estudiados están compuestos en su mayoría por los ácidos esteárico, palmítico y oleico. Estos ácidos representan el $88 \%$ y el $79 \%$, en el caso de los residuos grasos de res y de cerdo respectivamente, lo cual es consistente con los datos reportados en la literatura (Badui, 1993).

\subsection{Efecto de los factores sobre la conversión}

El análisis estadístico (Figura 1) demostró que todos los factores (radio molar de sustratos, biocatalizador y la presencia de solvente) son significativos sobre la conversión final. Además la interacción entre el biocatalizador y el radio molar de sustratos es igualmente importante en el desempeño de la reacción. Esto implicaría que todos los factores y la interacción que resultó significativa se deben optimizar.

En el sistema libre de solvente, las condiciones bajo las cuales se obtuvieron mayores conversiones fueron un radio molar 1:4 con la enzima N435, alcanzando una conversión del $79 \%$ en 48 horas. No obstante, en las otras condiciones de reacción analizadas se obtuvieron conversiones menores al $10 \%$ (Figura 2). Lee et al. (2002), en un estudio similar llevan a cabo la reacción de transesterificación con fracciones grasas animales y metanol con un radio molar $1: 3$ y $10 \% \mathrm{p} / \mathrm{v}$ de la enzima N435 logrando una conversión de apenas $2.8 \%$ después de 48 horas de reacción a una temperatura de $30^{\circ} \mathrm{C}$, sin embargo al agregar $10 \% \mathrm{p} / \mathrm{v}$ de sílica gel alcanzaron conversiones del $58 \%$. En el mismo estudio al utilizar etanol en una reacción con adicio-

Tabla 2

Perfil de ácidos grasos

\begin{tabular}{|c|c|c|c|c|c|}
\hline \multirow{2}{*}{ Ácido graso } & \multirow{2}{*}{ Tipo } & \multicolumn{2}{|c|}{ Pérfil obtenido (\%) } & \multicolumn{2}{|c|}{ Literatura (\%) $^{a}$} \\
\hline & & Grasa de cerdo & Grasa de res & Grasa de cerdo & Grasa de res \\
\hline Laúrico & Saturado & 0.08 & 0.08 & 0.10 & 0.10 \\
\hline Mirístico & Saturado & 1.46 & 2.83 & 1.50 & 3.20 \\
\hline Palmítico & Saturado & 26.85 & 28.64 & 26.00 & 24.30 \\
\hline Palmitoleico & Insaturado & 1.79 & 1.14 & 3.30 & 3.70 \\
\hline Heptadecanoico & Saturado & 0.43 & 2.00 & 0.40 & 1.50 \\
\hline Esteárico & Saturado & 18.36 & 33.13 & 13.50 & 18.60 \\
\hline Oleico & Insaturado & 33.72 & 26.45 & 43.90 & 42.60 \\
\hline Cis-vaccénico & Insaturado & 2.21 & 0.87 & 0.00 & 0.00 \\
\hline Linoleico & Insaturado & 12.17 & 1.89 & 9.50 & 2.60 \\
\hline Alfa-linolénico & Insaturado & 0.54 & 0.52 & 0.40 & 0.70 \\
\hline Araquídico & Saturado & 0.24 & 0.43 & 0.20 & 0.20 \\
\hline Eicosenoico & Insaturado & 0.67 & 0.10 & 0.70 & 0.30 \\
\hline Otros & & 1.48 & 1.92 & 0.50 & 2.20 \\
\hline Total & & 100.00 & 100.00 & 100.00 & 100.00 \\
\hline
\end{tabular}

${ }^{a}$ Datos de literatura: Badui (1993). 


\section{Diagrama de Pareto para \% Conv.}

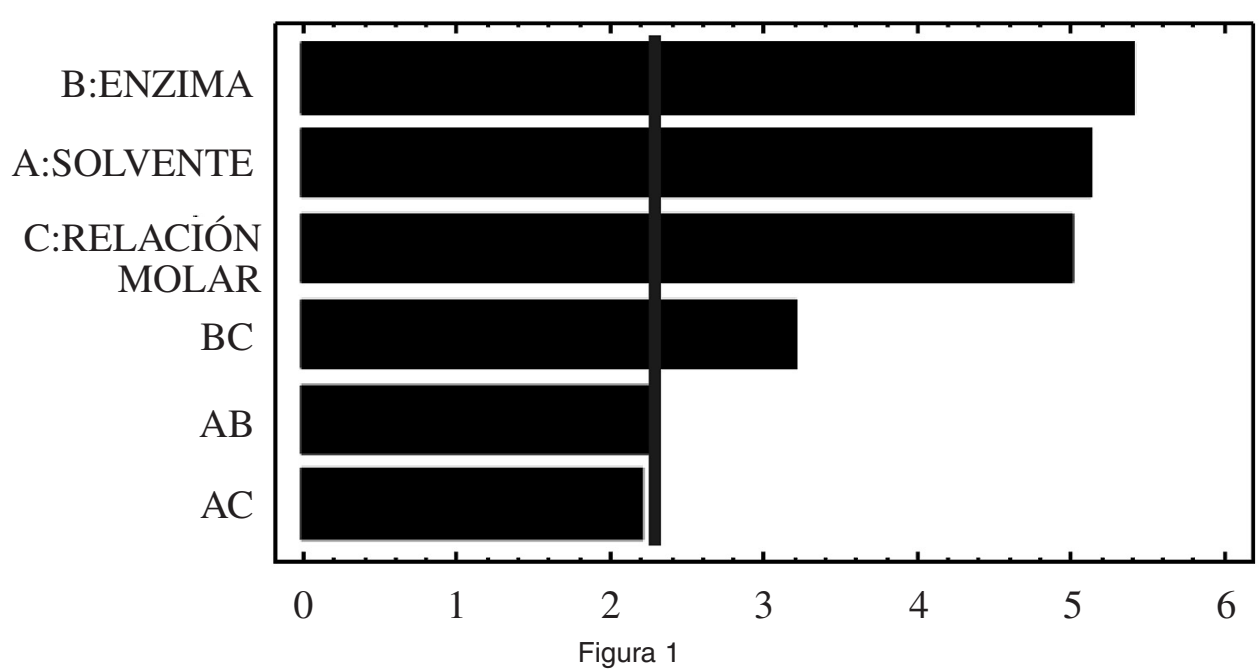

Diagrama de Pareto, análisis estadístico del \% de conversión. Los tres factores estudiados fueron: enzima, tipo de sistema y relación molar. Los tres factores fueron significativos con 95\% de confianza.

nes del equivalente a un radio molar durante 3 ciclos se logro una conversión del $70 \%$. Es decir que en el presente estudio se alcanzan mayores conversiones que las anteriormente reportadas para la reacción de transesterificación de grasas animales catalizada por lipasas inmovilizadas.

Algunos de los esfuerzos realizados para alcanzar las mejores condiciones de reacción como los de Dizge y Keskinler (2008) y Yagiz et al. (2007), utilizando aceites de desecho y aceite de cocina con la enzima TL IM de Thermomyces lanuginosa y los de Hsu et al. (2003), con grasas de restaurantes y la lipasa inmovilizada de Pseudomonas cepacia, han identificado, al igual que en el presente trabajo, que el radio molar es un parámetro importante en este tipo de reacciones, sobre todo el caso del sistema libre de solvente (Sandoval et al., 2002). Además el exceso moderado de alcohol,

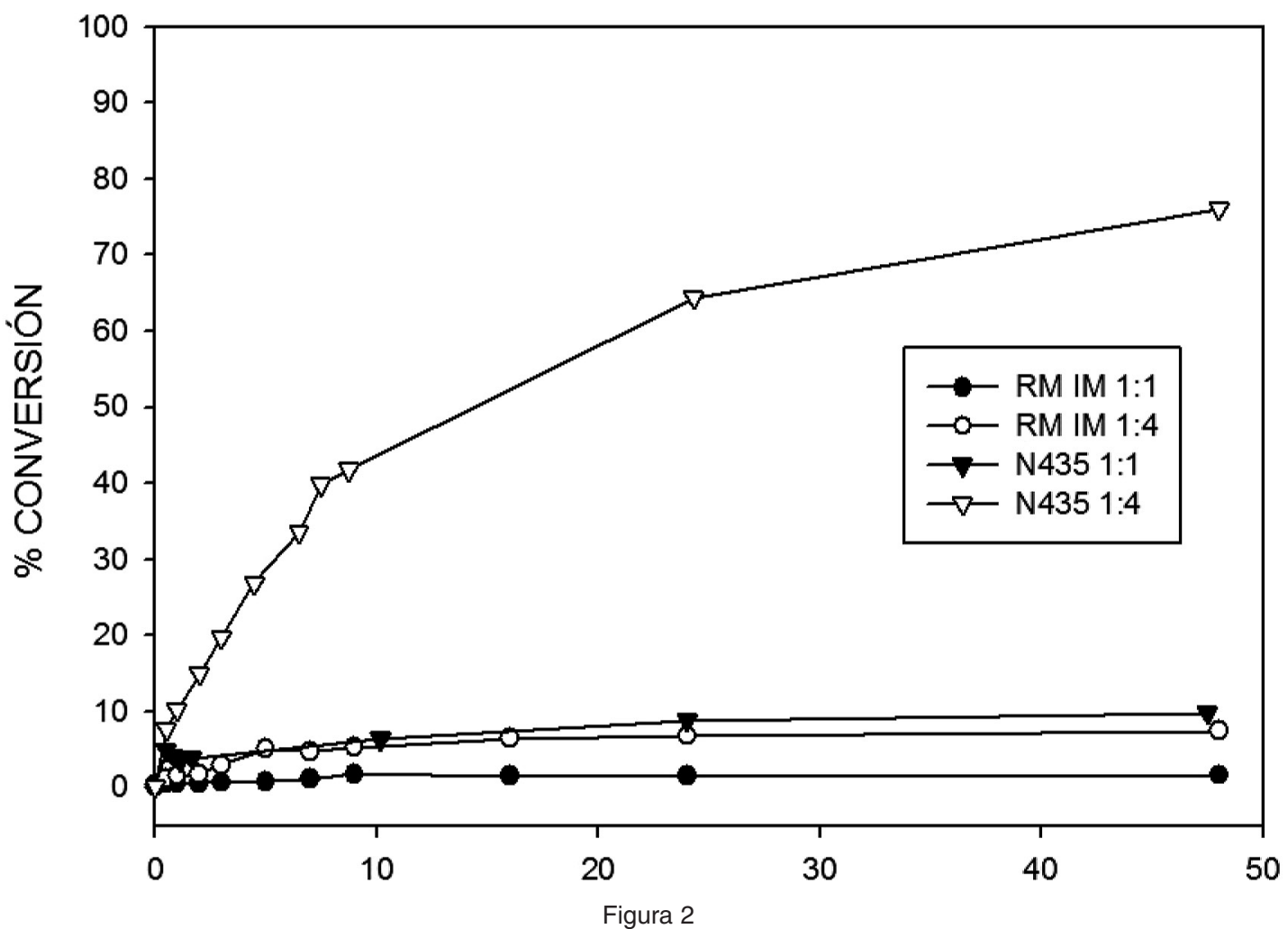

Efecto del radio molar triglicéridos: etanol en la reacción de transesterificación en un sistema libre de solvente, catalizada por lipasas inmovilizada de R. miehei (RM IM) y C. antarctica B (N435). Condiciones: $45^{\circ} \mathrm{C}, 100 \mathrm{mg}$ de enzima, $300 \mathrm{rpm}$. 
ayuda a alcanzar mayores conversiones de reacción, probablemente debido a que permite que baje la viscosidad y los sustratos sean más accesibles al sitio activo de la enzima. Sin embargo también es conocido que el exceso de etanol o metanol es un inhibidor de las lipasas (Sandoval et al., 2001).

Como se puede observar en la Figura 3, al igual que en el sistema libre de solvente en presencia del solvente terbutanol, las mejores condiciones de reacción fueron con un radio molar 1:4 y la enzima N435. Sin embargo, a diferencia del sistema sin solvente, las conversiones obtenidas con terbutanol para la enzima RM IM fueron mayores alcanzando conversiones de $30 \%$ y $35 \%$ con relación molar $1: 1$ y 1:4 respectivamente. Sin embargo, en el caso de la enzima N435 las conversiones fueron menores en terbutanol, $50 \%$ y $65 \%$ con las mismas relaciones anteriores. Además en terbutanol el equilibrio se alcanza en un menor tiempo, por lo que las cinéticas sólo se siguieron durante 24 horas.

En la mayoría de estudios similares donde se llevan a cabo reacciones de esterificación-transesterificación con solventes, se utiliza hexano debido a la estabilidad de las lipasas en este solvente (Chulalaksananukul et al., 1990; Guncheva y Zhiryakova, 2008; Sandoval et al., 2002). A diferencia de estos trabajos, en nuestro caso se eligió el terbutanol, ya que es más seguro que el hexano, pues es menos tóxico y menos volátil. Además, en estudios preliminares de transesterificación de aceite de oliva con etanol, se observó mayor velo- cidad de reacción y conversión en terbutanol que en hexano (los datos no se presentan). Los mejores resultados de la transesterificación en terbutanol comparado con hexano pueden explicarse debido a que en este tipo de solventes más polares, el coeficiente de actividad termodinámica del alcohol, que además de ser sustrato es también inhibidor competitivo de la lipasa, disminuye; disminuyendo su disponibilidad real y volviéndose así menos inhibidor, además de tener una actividad termodinámica menor en el denominador de la constante de equilibrio (Sandoval et al., 2002).

\subsection{Efecto de los factores sobre la velocidad inicial de reacción}

Las velocidades iniciales de cada cinética, se muestran en la Tabla 3. El único factor importante sobre la velocidad inicial de reacción es el tipo de biocatalizador utilizado (Figura 4). La enzima N435 también se destaca en este aspecto, alcanzando una velocidad inicial aproximadamente 3 veces mayor que la de la enzima RM IM.

Las velocidades iniciales de reacción son uno de los parámetros importantes para llevar a cabo los estudios de la reacción. Sandoval et al. (2002) compararon las velocidades iniciales en un sistema por lotes para la esterificación de ácido oleico con etanol catalizada por la lipasa inmovilizada RM IM, en un sistema libre de solvente y utilizando hexano como solvente. Sus resultados mostraron que la

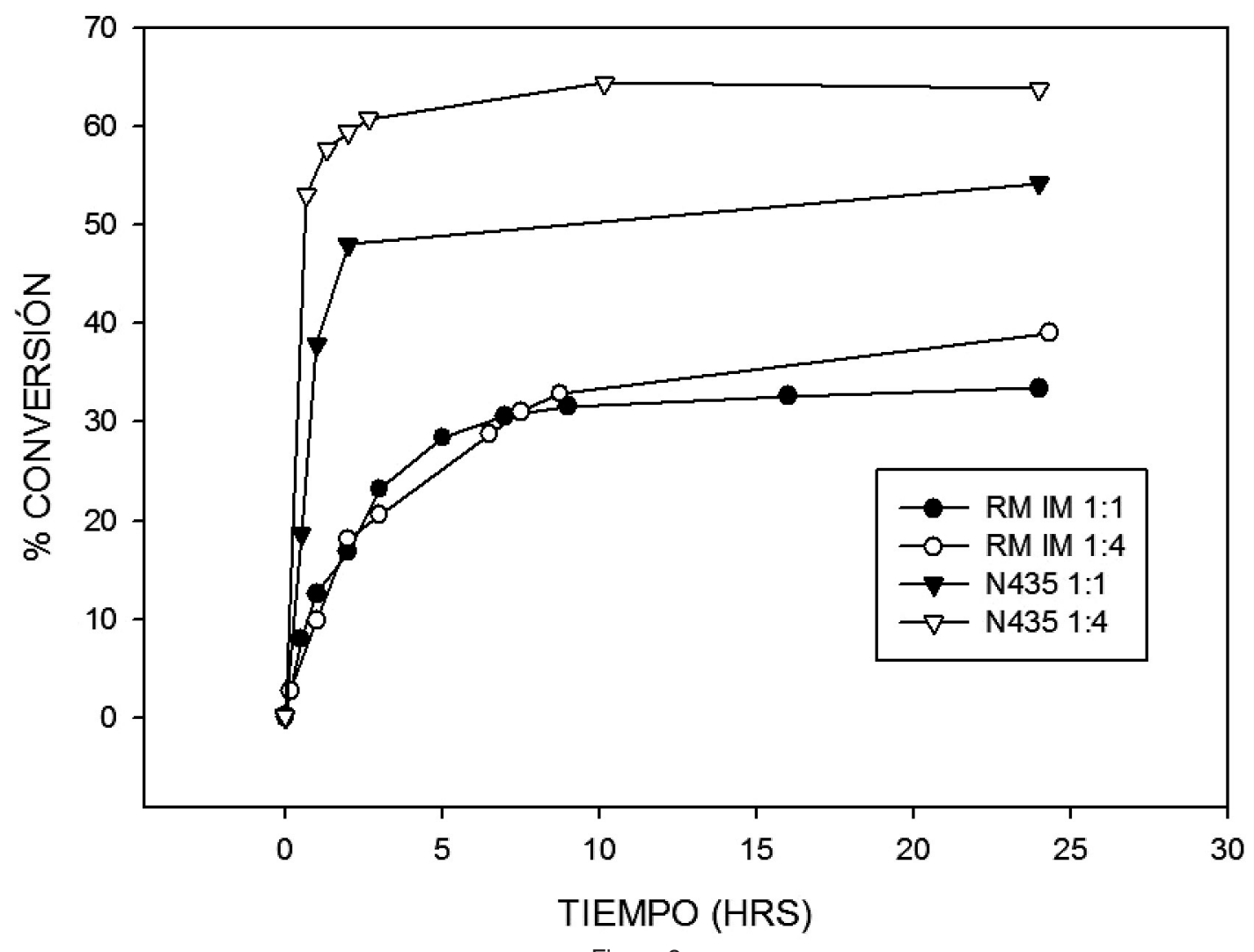

Figura 3

Efecto del radio molar triglicéridos: etanol en la reacción de transesterificación empleando terbutanol como solvente, catalizada por lipasas inmovilizada de R. miehei (RM IM) y C. antartica B (N435). Condiciones: $45^{\circ} \mathrm{C}, 100 \mathrm{mg}$ de enzima, $300 \mathrm{rpm}$. 
Tabla 3

Velocidades iniciales de reacción

\begin{tabular}{lccc}
\hline \multirow{2}{*}{ Enzima } & Relación molar grasa: etanol & \multicolumn{2}{c}{ Vi (mmol/h/g de enzima) } \\
\cline { 3 - 4 } & & Libre de solvente & Terbutanol \\
\hline \multirow{2}{*}{ N 435} & $1: 1$ & 10.7 & 10.3 \\
RM IM & $1: 4$ & 11.2 & 13.3 \\
& $1: 1$ & 0.5 & 2.9 \\
\hline
\end{tabular}

velocidad inicial de la reacción es independiente de la presencia del solvente y se ve afectado principalmente por el radio molar, sin embargo en nuestro caso al probar dos enzimas diferentes el efecto principal esta dado por este factor. Es comprensible la mayor velocidad obtenida al utilizar la lipasa N435 ya que se trata de una lipasa no selectiva hacia las tres posiciones del triglicérido, mientras que la lipasa RM IM es 1,3 regioselectiva (Schmid and Verger, 1998), por lo que la transesterificación sólo se completa después de que se realiza la migración intramolecular del acilo de la posición 2 a una de las otras posiciones.

\section{CONCLUSIONES}

Los residuos grasos animales cuentan con una gran cantidad de ácidos grasos esterificables que los hacen ideales para la producción de biodiesel y disminuye los costos de producción de este biocombustible. Además permite la solución de los problemas ambientales asociados con la generación de productos secundarios de la industria ganadera.

Los residuos grasos animales son una materia prima viable para las reacciones de transesterifica- ción por vía enzimática. La enzima N435 fue la mejor opción en el sistema libre de solvente y en el sistema utilizando terbutanol como solvente, demostrando su versatilidad, la cual es debida en parte a su capacidad para modificar las tres cadenas del triglicérido de forma indistinta, así como al tipo de soporte en el que se encuentra inmovilizada la lipasa.

Para llevar a cabo con éxito la síntesis de biodiesel, es determinante la selección de los parámetros adecuados como son: el catalizador, el radio molar y el tipo de solvente a utilizar. Estos tres parámetros demostraron ser claves para llevar a cabo la transesterificación.

Por otro lado en el sistema libre de solvente a pesar de que se obtiene mayor conversión, es necesario mayor tiempo de reacción, lo cual se debe en gran medida a las limitaciones difusionales propias del sistema. Sin embargo a nivel industrial tiene la ventaja de que se eliminarían los costos de recuperación del solvente.

\section{AGRADECIMIENTOS}

El presente trabajo fue realizado gracias a la financiación del proyecto SLP2005-01-20 del

\section{Diagrama de Pareto para Vi}

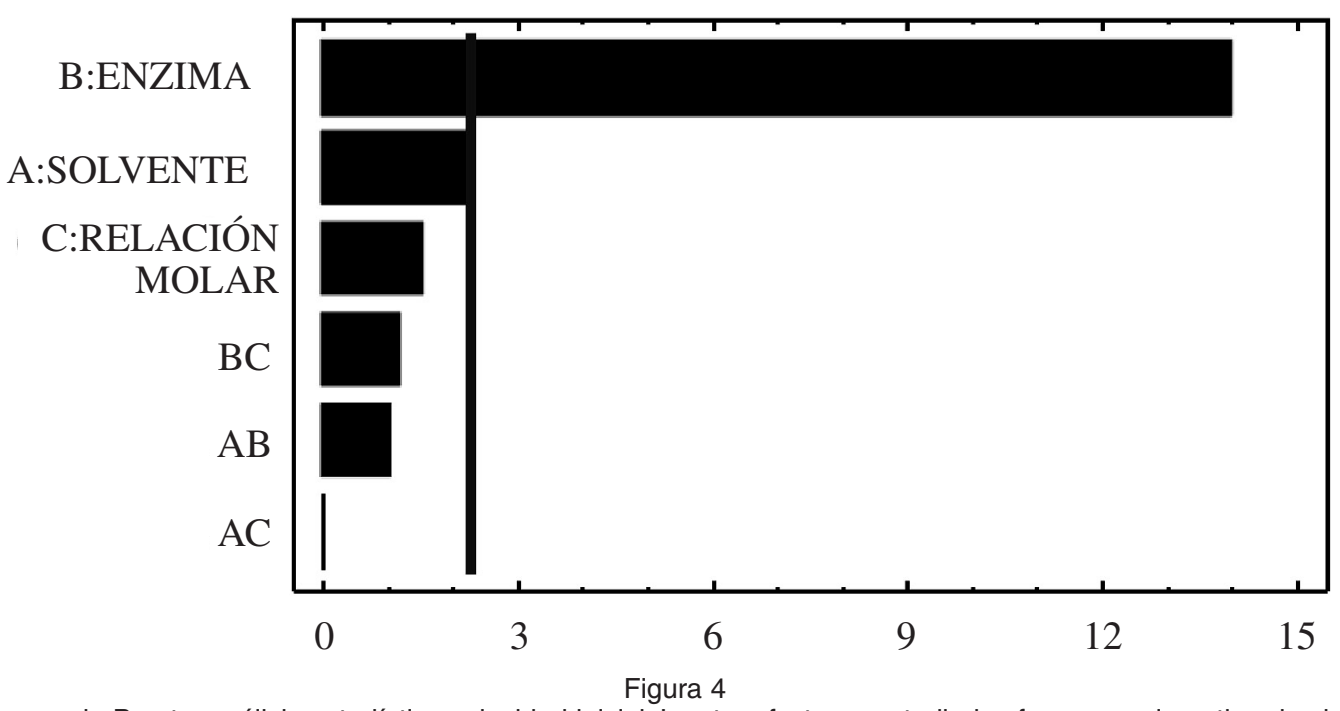

Diagrama de Pareto, análisis estadístico velocidad inicial. Los tres factores estudiados fueron: enzima, tipo de sistema y relación molar. El único factor significativo fue la enzima con $95 \%$ de confianza. 
Fondo Mixto CONACYT-Gobierno de San Luis Potosí (México). Ivanna Rivera agradece al CONACYT su beca de maestría.

\section{REFERENCIAS}

Al-Zuhair S. 2005. Production of biodiesel by lipasecatalyzed transesterification of vegetable oils: A kinetics study. Biotechnol Progress 21, 1442-1448.

AOAC International. 1984. Official methods of analysis. Peroxide value: $28.022-28.023$.

AOAC International. 1989. Official methods of analysis. Free fatty acid: Ca $5 \mathrm{a}-40$.

Badui Dergal S. 1993. Química de los alimentos. Tercera edición. Pearson Educación. 213-232. Naucalpan Edo. México. México.

Balat M, Balat H. 2008. A critical review of bio-diesel as a vehicular fuel Energy Convers Manage 49, 27272741.

Canakci M. 2005. The potential of restaurant waste lipids as biodiesel feedstocks. Bioresour Technol. 11, 1-8.

Canakci M, Sanli H. 2008. Biodiesel production from various feedstocks and their effects on the fuel properties. J. Ind. Microbiol \& Biotechno/ 35, 431-441.

Chulalaksananukul W, Condoret JS, Delorme P, Willemot RM. 1990. kinetic-study of esterification by immobilized lipase in n-hexane. Febs. Letters 276, 181-184.

Dizge N, Keskinler B. 2008. Enzymatic production of biodiesel from canola oil using immobilized lipase. Biomass Bioenergy 32, 1274-1278.

Du W. 2005. Study on acyl migration in immobilized lipozyme TL- catalized transesterificación of soybean oil for biodiesel production. J. Mol. Catal. B. Enzymatic 37, 68-71.
Guncheva MH, Zhiryakova D. 2008. High-yield synthesis of wax esters catalysed by modified Candida rugosa lipase. Biotechnol Lett. 30, 509-512.

Haas MJ, McAloon AJ, Yee WC, Foglia TA. 2006. A process model to estimate biodiesel production cost. Bioresour Technol. 97,671-678.

Hsu AF, Jones KC, Foglia TA, Marmer WN. 2003. Optimization of alkyl ester production from grease using a phyllosilicate sol-gel immobilized lipase. Biotechnol Lett. 25, 1713-1716.

Knothe G, Dunn RO, O. BM. 1996. Technical aspects of biodiesel standards. Inform. 7, 827-829

Lee T, Foglia T, Chang KS. 2002. Production of alkyl ester as biodiesel from fractionated lard and restaurant grease. JAOCS 79, 191-195.

Meher LC, Sagar DV, Naik SN. 2006. Technical aspects of biodiesel production by transesterification - a review. Renew Sustain Energy Rev. 10, 248-268.

Royon D, Daz M, Ellenrieder G, Locatelli S. 2007. Enzymatic production of biodiesel from cotton seed oil using t-butanol as a solvent. Bioresour Technol. 98, 648-653.

Sandoval G, Condoret JS, Marty A. 2001. Thermodynamic Activity Based Enzyme Kinetics: an efficient tool for nonaqueous enzymology. AlChE J. 47, 718-726.

Sandoval G, Monsan P, Marty A. 2002. Esterification by immobilized lipases: kinetic and thermodynamic arguments. Biotechnol Bioeng. 78, 313-320.

Schmid RD, Verger R. 1998. Lipases: Interfacial Enzymes with Attractive Applications. Angrew. Chem. Int. ed. 37, 1608-1633

Yagiz F, Kazan D, Akin AN. 2007. Biodiesel production from waste oils by using lipase immobilized on hydrotalcite and zeolites. J. Chem. Eng. 134, 262-267

Recibido: 25/2/09 Aceptado: 2/4/09 